\title{
BOREL ACTIONS OF POLISH GROUPS
}

\author{
HOWARD BECKER AND ALEXANDER S. KECHRIS
}

\begin{abstract}
We show that a Borel action of a Polish group on a standard Borel space is Borel isomorphic to a continuous action of the group on a Polish space, and we apply this result to three aspects of the theory of Borel actions of Polish groups: universal actions, invariant probability measures, and the Topological Vaught Conjecture. We establish the existence of universal actions for any given Polish group, extending a result of Mackey and Varadarajan for the locally compact case. We prove an analog of Tarski's theorem on paradoxical decompositions by showing that the existence of an invariant Borel probability measure is equivalent to the nonexistence of paradoxical decompositions with countably many Borel pieces. We show that various natural versions of the Topological Vaught Conjecture are equivalent with each other and, in the case of the group of permutations of $\mathbb{N}$, with the model-theoretic Vaught Conjecture for infinitary logic; this depends on our identification of the universal action for that group.
\end{abstract}

A Polish space (group) is a separable, completely metrizable topological space (group). A standard Borel space is a Polish space with the associated Borel structure. A Borel action of a Polish group $G$ on a standard Borel space $X$ is an action $(g, x) \in G \times X \mapsto g \cdot x$ of $G$ on $X$ which is Borel, as a function from the space $G \times X$ into $X$. The structure of Borel actions of Polish locally compact, i.e., second countable locally compact, topological groups has long been studied in ergodic theory, operator algebras, and group representation theory. See, for example, [AM, Zi, Sin, VF, Mo, Ma1-Ma3, G, Var, FHM, Ra1, Ra2, K] for a sample of works related to the themes that we will be studying here. More recently, there has been increasing interest in the study of Borel actions of nonlocally compact Polish groups. One instance is the Vaught Conjecture, a well-known open problem in mathematical logic and the Topological Vaught Conjecture (cf. $\S \S 1,2$ ) (see, e.g., [Vau, Mi, St, Sa, L, Be, BM]). Another is the ergodic theory and unitary group representation theory of so-called "large groups" (see, e.g., [Ve, O]). Also [E] is relevant here.

Our purpose in this note is to announce a number of results about Borel actions of general Polish groups. With the exception of Theorem 2.1, these results are new even for locally compact groups. Our Theorem 2.1 is known in the locally compact case [Ma2, Var], but the proofs in this case relied on Haar measure, so our proof seems new even in this case. The fundamental result is Theorem 1.1, stating that for the actions considered Borel actions are equivalent to continuous ones.

The paper is divided into three parts. The first part deals with Theorem 1.1 and a related result solving problems of Ramsay [Ra2] (raised in the locally

Received by the editors April 16, 1992 and, in revised form, October 15, 1992.

1991 Mathematics Subject Classification. Primary 03E15, $28 \mathrm{D} 15$.

The first author's research was partially supported by NSF Grant DMS-8914426. The second author's research was partially supported by NSF Grant DMS-9020153. 
compact case) and Miller [Mi] and, as a direct application, shows the equivalence of three possible versions of the Topological Vaught Conjecture. In the second part, we establish the existence of universal Borel actions for any Polish group, extending a result of Mackey [Ma2] and Varadarajan [Var] from the locally compact case. This is also applied to establish the equivalence of the Topological Vaught Conjecture for the symmetric group $S_{\infty}$, i.e., the permutation group of $\mathbb{N}$, with the usual model-theoretic Vaught Conjecture for $L_{\omega_{1} \omega}$. The final section deals with the problem of existence of invariant (countably additive) Borel probability measures for a Borel action of a Polish group. It is a well-known theorem of Tarski (see [Wn]) that an arbitrary action of a group $G$ on a set $X$ admits a finitely additive invariant probability measure defined on all subsets of $X$ iff there is no "paradoxical decomposition" of $X$ with finitely many pieces. We show that there is a complete analog of Tarski's theorem for Borel actions of Polish groups and countably additive Borel invariant probability measures when we allow "paradoxical decompositions" to involve countably many Borel pieces. Our proof uses the results of $\S 1$ and the basic work of Nadkarni [N], who proves this result in case $G=\mathbb{Z}$.

\section{BOREL VS. TOPOLOGICAL GROUP ACTIONS}

By a Borel $G$-space we mean a triple $(X, G, \alpha)$, where $X$ is a standard Borel space, $G$ a Polish group, and $\alpha: G \times X \rightarrow X$ a Borel action of $G$ on $X$. We will usually write $X$ instead of $(X, G, \alpha)$ and $\alpha(g, x)=g \cdot x$, when there is no danger of confusion. Two Borel $G$-spaces $X, Y$ are Borel isomorphic if there is a Borel bijection $\pi: X \rightarrow Y$ with $\pi(g \cdot x)=g \cdot \pi(x)$. A Polish G-space consists of a triple $(X, G, \alpha)$, where $X$ is a Polish space and $\alpha: G \times X \rightarrow X$ is continuous (thus any Polish $G$-space is also Borel).

Theorem 1.1. Let $X$ be any Borel $G$-space. Then there is a Polish G-space $Y$ Borel isomorphic to $X$.

This answers a question of [Ra2] (for locally compact $G$ ) and [Mi]. Theorem 1.1 was known classically for discrete $G$ and was proved in [Wh] for $G=\mathbb{R}$. A convenient reformulation of our result is the following: If $X$ is a Borel $G$ space, then there is a Polish topology $\tau$ on $X$ giving the same Borel structure for which the action becomes continuous. In our proof, sketched in $\S 4$, we define $\tau$ explicitly and use a criterion of Choquet to show that it is a Polish topology. One can also prove a version of Theorem 1.1 for more general "definable" actions of $G$ on separable metrizable spaces.

It is a classical result of descriptive set theory (see, e.g., [Ku]) that for any Polish space $X$ and any Borel set $B \subseteq X$, there is a Polish topology, finer than the topology of $X$, and thus having the same Borel structure as $X$, in which $B$ is clopen. We extend this result in the case of Polish $G$-actions. The result below was known for $S_{\infty}$ (see [Sa]) and is essentially a classical result for discrete $G$.

Theorem 1.2. Let $X$ be a Polish G-space and $B \subseteq X$ an invariant Borel set. Then there is a Polish topology finer than the topology of $X$ (and thus having the same Borel structure) in which $B$ is now clopen and the action is still continuous.

The Topological Vaught Conjecture for a Polish group $G$, first conjectured by Miller (see, e.g., [Ro, p. 484]) has been usually formulated in one of three, a 
priori distinct, forms. Let:

$\operatorname{TVC}_{I}(G) \Leftrightarrow$ For any Polish $G$-space $X$ and any invariant Borel set $B \subseteq X$, either $B$ contains countably many orbits or else there is a perfect set $P \subseteq B$ with any two distinct members of $P$ belonging to different orbits;

$\operatorname{TVC}_{I I}(G) \Leftrightarrow$ Same as $\operatorname{TVC}_{I}(G)$ but with $B=X$;

$\operatorname{TVC}_{I I I}(G) \Leftrightarrow$ For any Borel $G$-space $X$ either $X$ contains countably many orbits or else there is an uncountable Borel set $P \subseteq X$ with any two distinct members of $P$ belonging to different orbits.

Clearly, $\operatorname{TVC}_{I I I}(G) \Rightarrow \mathrm{TVC}_{I}(G)$, since a Borel set in a Polish space is uncountable iff it contains a perfect set, and $\mathrm{TVC}_{I}(G) \Rightarrow \mathrm{TVC}_{I I}(G)$. From Theorem 1.1 we have $\operatorname{TVC}_{I I}(G) \Rightarrow \operatorname{TVC}_{I I I}(G)$, thus

Corollary 1.3. For any Polish group $G$, all three forms of the Topological Vaught Conjecture for $G$ are equivalent.

The Topological Vaught Conjecture was motivated by the Vaught Conjecture in logic, which we discuss in $\S 2$. The truth or falsity of $\operatorname{TVC}(G)$ remains open for general Polish $G$. It has been proved for $G$ locally compact (see [Sil]) or abelian (see [Sa]). It can be also shown, using a method of Mackey, that if $G$ is a closed subgroup of $H$, then $\operatorname{TVC}(H)$ implies $\operatorname{TVC}(G)$. Of particular interest is the case $G=S_{\infty}$ (see $\S 2$ ). It is known (see [Bu]) that for any Polish $G$-space $X$ there are either $\leq \aleph_{1}$ many orbits or else perfectly many orbits, i.e., there is a perfect set with any two distinct members of it belonging to different orbits. So assuming the negation of the Continuum Hypothesis $(\mathrm{CH})$, there are $2^{\aleph_{0}}$ many orbits iff there are perfectly many orbits. Thus the meaning of TVC is that the set of orbits cannot be a counterexample to $\mathrm{CH}$.

\section{UNIVERSAL ACTIONS}

Let $X, Y$ be Borel $G$-spaces. A Borel embedding of $X$ into $Y$ is a Borel injection $\pi: X \rightarrow Y$ such that $\pi(g \cdot x)=g \cdot \pi(x)$. Note that $\pi[X]$ is an invariant Borel subset of $Y$. By the usual Schroeder-Bernstein argument, $X, Y$ can be Borel-embedded in each other iff they are Borel isomorphic. A Borel $G$-space $\mathscr{U}$ is universal if every Borel $G$-space $X$ can be Borel-embedded into $\mathscr{U}$. It is unique up to Borel isomorphism.

Theorem 2.1. For any Polish group $G$, there is a universal Borel G-space $\mathscr{U}_{G}$. Moreover, $\mathscr{U}_{G}$ can be taken to be a Polish G-space.

Actually the proof of Theorem 2.1 shows that one can Borel-embed in $\mathscr{U}_{G}$ any Borel action of $G$ on a separable metrizable space. In particular, a Borel action of $G$ on an analytic Borel space (i.e., an analytic set with its associated Borel structure) is Borel isomorphic to a continuous action of $G$ on an analytic space, i.e., an analytic set with its associated topology. For locally compact $G$, Theorem 2.1 has been proved in [Ma2, Var]. In this case, $\mathscr{U}_{G}$ can be taken to be compact. Our proof gives a new proof of this result, with a different universal space, which avoids using the Haar measure. It is unknown whether $\mathscr{U}_{G}$ can be taken to be compact in the general case.

In the particular case of the group $G=S_{\infty}$, with the Polish topology it inherits as a $G_{\delta}$ subspace of the Baire space $\mathbb{N}^{\mathbb{N}}$, our proof of Theorem 2.1 gives a particularly simple form of $\mathscr{U}_{G}$, which we use below. Let $X_{\infty}$ be the space of all maps from the set $\mathbb{N}^{<\mathbb{N}}$ of finite sequences of natural numbers into $2=\{0,1\}$. 
Clearly this space is homeomorphic to the Cantor space. Consider the following action of $S_{\infty}$ on $X_{\infty}: g \cdot x\left(s_{0}, s_{1}, \ldots, s_{n-1}\right)=x\left(g^{-1}\left(s_{0}\right), \ldots, g^{-1}\left(s_{n-1}\right)\right)$, if $s=\left(s_{0}, s_{1}, \ldots, s_{n-1}\right) \in \mathbb{N}^{<\mathbb{N}}$. Then $X_{\infty}$ with this action is a universal $S_{\infty}$ space and is clearly compact. We would like to thank R. Dougherty for suggesting that we can use the above space instead of a somewhat more complicated one we had originally.

This leads to an interesting fact concerning the Vaught Conjecture. The Vaught Conjecture for $L_{\omega_{1} \omega}$ is the assertion that every $L_{\omega_{1} \omega}$ sentence in a countable language has either countably many or else perfectly many countable models, up to isomorphism. Let $L$ be a countable language which we assume to be relational, say $L=\left\{R_{i}\right\}_{i \in I}$, where $I$ is a countable set and $R_{i}$ is a $n_{i}$-ary relation symbol. Let $X_{L}=\prod_{i \in I} 2^{\mathbb{N}^{n_{i}}}$ which is homeomorphic to the Cantor space (if $L \neq \varnothing$ ). We view $X_{L}$ as the space of countably infinite structures for $L$, identifying $x=\left(x_{i}\right)_{i \in I} \in X_{L}$ with the structure $\mathscr{A}=\langle\mathbb{N}$, $\left.R_{i}^{\mathscr{A}}\right\rangle$, where $R_{i}^{\mathscr{A}}(s) \Leftrightarrow x_{i}(s)=1$. The group $S_{\infty}$ acts in the obvious way on $X_{L}: g \cdot x=y \Leftrightarrow \forall i\left[y_{i}\left(s_{0}, \ldots, s_{n_{i}-1}\right)=1\right.$ iff $\left.x_{i}\left(g^{-1}\left(s_{0}\right), \ldots, g^{-1}\left(s_{n_{i}-1}\right)\right)=1\right]$. Thus if $x, y$ are identified with the structures $\mathscr{A}, \mathscr{B}$ resp., $g . x=y$ iff $g$ is an isomorphism of $\mathscr{A}, \mathscr{B}$. This action is called the logic action of $S_{\infty}$ on $X_{L}$. By [L-E] the Borel invariant subsets of this action are exactly the sets of models of $L_{\omega_{1} \omega}$ sentences. So the Vaught Conjecture for $L_{\omega_{1} \omega}$ (VC), a notorious open problem, is the assertion that for any countable $L$ and any Borel invariant subset $B \subseteq X_{L}$, either $B$ contains countably many orbits or else there is a perfect set $P \subseteq B$, no two distinct members of which are in the same orbit. It is thus a special case of the Topological Vaught Conjecture for $S_{\infty}$. (Historically, of course, TVC came much later and was inspired by VC.) The universal Borel $S_{\infty}$-space $X_{\infty}$ above is the same as $X_{L_{\infty}}$ for $L_{\infty}=\left\{R_{n}\right\}_{n \in \mathbb{N}}$, with $R_{n}$ an $n$-ary relation, so we have

Corollary 2.2. The logic action on the space of structures of the language containing an $n$-ary relation symbol for each $n \in \mathbb{N}$ is a universal $S_{\infty}$-space. In particular, Vaught's conjecture for $L_{\omega_{1} \omega}$ is equivalent to the Topological Vaught Conjecture for $S_{\infty}$.

We conclude with an application to equivalence relations. Given two equivalence relations $E, F$ on standard Borel spaces $X, Y$ resp., we say that $E$ is Borel embeddable in $F$ iff there is a Borel injection $f: X \rightarrow Y$ with $x E y \Leftrightarrow f(x) F f(y)$. Given a class of equivalence relations $\mathscr{S}$, a member $F \in \mathscr{S}$ is called universal if every $E \in \mathscr{S}$ is Borel embeddable in $F$. For each Borel $G$-space denote by $E_{G}$ the corresponding equivalence relation induced by the orbits of the action: $x E_{G} y \Leftrightarrow \exists g \in G(g \cdot x=y)$. The following is based on the proof of Theorem 2.1 and the work in [U], a paper which was brought to our attention by $\mathrm{W}$. Comfort.

Corollary 2.3. There is a universal equivalence relation in the class of equivalence relations $E_{G}$ induced by Borel actions of Polish groups.

\section{INVARIANT MEASURES}

Let $G$ be a group acting on a set $X$. Given $A, B \subseteq X$, we say that $A, B$ are equivalent by finite decomposition, in symbols $A \sim B$, if there are partitions $A=\bigcup_{i=1}^{n} A_{i}, B=\bigcup_{i=1}^{n} B_{i}$, and $g_{1}, \ldots, g_{n}$ with $g_{i} . A_{i}=B_{i}$. We say that $X$ 
is $G$-paradoxical if $X \sim A \sim B$ with $A \cap B=\varnothing$. A finitely additive probability (f.a.p.) measure on $X$ is a map $\varphi: \mathscr{P}(X) \rightarrow[0,1]$ such that $\varphi(X)=1$, $\varphi(A)+\varphi(B)=\varphi(A \cup B)$, if $A \cap B=\varnothing$. Such a $\varphi$ is $G$-invariant if $\varphi(A)=$ $\varphi(g . A)$ for all $g \in G$ and $A \subseteq X$. If $X$ is $G$-paradoxical, there can be no $G$-invariant f.a.p. measure on $X$. A well-known theorem of Tarski asserts that the converse is also true (see [Wn]).

Theorem 3.1 (Tarski). Let a group $G$ act on a set $X$. Then there is a $G$-invariant finitely additive probability measure on $X$ iff $X$ is not $G$-paradoxical.

It is natural to consider to what extent Tarski's theorem goes through for countably additive probability measures. Let $(X, \mathscr{A})$ be a measurable space, i.e., a set equipped with a $\sigma$-algebra. Let a group $G$ act on $X$ so that $A \in \mathscr{A} \Rightarrow$ g. $A \in \mathscr{A}$. Given $A, B \in \mathscr{A}$, we say that $A, B$ are equivalent by countable decomposition, in symbols $A \sim_{\infty} B$, if there are partitions $A=\bigcup_{i \in I} A_{i}, B=$ $\bigcup_{i \in I} B_{i}$, with $I$ countable and $A_{i}, B_{i} \in \mathscr{A}$, and $\left\{g_{i}\right\}_{i \in I}$ so that $g_{i} \cdot A_{i}=B_{i}$. We say that $(X, \mathscr{A})$, or just $X$ if there is no danger of confusion, is countably $G$-paradoxical if $X \sim_{\infty} A \sim_{\infty} B$ with $A, B \in \mathscr{A}$ and $A \cap B=\varnothing$. A probability measure $\mu$ on $(X, \mathscr{A})$ is $G$-invariant if $\mu(A)=\mu(g \cdot A)$ for all $g \in G$ and $A \in \mathscr{A}$. Again, if there is a $G$-invariant probability measure on $(X, \mathscr{A}), X$ cannot be countably $G$-paradoxical. Is the converse true? It turns out that the answer is negative in this generality. See [Wn, Za] for more on the history and some recent developments on this problem. We show here, however, that the converse holds in most regular situations, i.e., when $G$ is Polish and acts in a Borel way on a standard Borel space $X$.

Theorem 3.2. Let $X$ be a Borel G-space. Then the following are equivalent:

(1) $X$ is not countably G-paradoxical;

(2) there is a $G$-invariant Borel probability measure on $X$.

The proof of this theorem is based on the results in $\S 1$ and [N], which proves Theorem 3.2 for the case $G=\mathbb{Z}$. It turns out that Theorem 3.2 holds also for any continuous action of a separable topological group on a Polish space $X$.

\section{SKetches of PROOFS}

For Theorem 1.1. We have a Borel action of $G$ on $X$. Fix a countable basis $\mathscr{B}$ for the topology of $G$. For $A \subseteq X$ and $U \subseteq G$ open, let $A^{\Delta U}=\{x: g \cdot x \in A$ for a set of $g$ 's which is nonmeager in $U\}$ be the Vaught transform [Vau]. We first find a countable Boolean algebra $\mathscr{C}$ of Borel subsets of $X$ such that (1) $A \in \mathscr{C} \Rightarrow A^{\Delta U} \in \mathscr{C}$ for $U \in \mathscr{B}$, and (2) the topology generated by $\mathscr{C}$ is Polish. Then let $\tau$ be the topology on $X$ generated by $\left\{A^{\Delta U}: A \in \mathscr{C}\right.$, $U \in \mathscr{B}\}$. It suffices to show that $\tau$ is a Polish topology and that the action of $G$ is continuous in this topology. Then since $\tau$ consists of Borel sets in $X$ and gives a Polish topology, it gives rise to the original Borel structure of $X$.

The continuity of the action may be checked by direct computation. To see that $\tau$ gives a Polish topology, we first check that it is $T_{1}$ and regular, as well as obviously second countable, hence metrizable, and then apply a criterion of Choquet to conclude that it is Polish. Choquet's criterion may be stated as follows.

Associate to $X$ the strong Choquet game, in which the first player specifies a sequence of open sets $U_{n}$ and elements $x_{n} \in U_{n}$, while the second player 
responds with open sets $V_{n}$, satisfying $x_{n} \in V_{n} \subseteq U_{n}$ and $U_{n+1} \subseteq V_{n}$; the second player wins if the intersection of the $U_{n}$ is nonempty. The space $X$ is called a strong Choquet space (see, e.g., [HKL]) if the second player has a winning strategy for this game, and Choquet's criterion (unpublished, but see [C] for a related version) states that a topological space is Polish if and only if it is separable, metrizable, and strong Choquet.

For Theorem 1.2. The proof is quite similar, taking a somewhat larger $\mathscr{C}$ and including a basis for the original topology in $\tau$.

For Theorem 2.1. Let $\mathscr{F}(G)$ be the space of closed subsets of $G$ with the (standard) Effros Borel structure, i.e., the one generated by the sets $\mathscr{F}_{V}=\{F \in$ $\mathscr{F}(G): F \cap V \neq \varnothing\}$, for $V \subseteq G$ open. Let $G$ act on $\mathscr{F}(G)$ by left multiplication and let $\mathscr{U}_{G}=\mathscr{F}(G)^{\mathbb{N}}$, with $G$ acting coordinatewise. Given any Borel $G$-space $X$, let $\left\{S_{n}\right\}$ be a sequence of Borel subsets of $X$ separating points. For each $A \subseteq G$, let $D(A)=\{g \in G$ : For every neighborhood $U$ of $g, A$ is nonmeager in $U\}$ and map $x \in X$ into the sequence $f(x)=\left\{D\left(\widetilde{S}_{n}\right)^{-1}\right\} \in \mathscr{U}_{G}$, where $\widetilde{S}_{n}=\left\{g: g \cdot x \in S_{n}\right\}$. This is an embedding of $X$ into $\mathscr{U}_{G}$. We can now make $\mathscr{U}_{G}$ into a Polish $G$-space using Theorem 1.1. When $G$ is locally compact and $\bar{G}=G \cup\{\infty\}$ is the one-point compactification of $G$, then we can extend the action of $G$ on itself by left multiplication to $\bar{G}$ by setting $g \cdot \infty=\infty$. Then instead of $\mathscr{F}(G)$ we can use $\mathscr{F}(\bar{G})=K(\bar{G})=$ the compact metrizable space of compact subsets of $\bar{G}$, with the obvious $G$-action, and the universal space is now $K(\bar{G})^{\mathbb{N}}$, which is compact. (Alternatively, we can use the Fell topology on $\mathscr{F}(G)$.)

For Corollary 2.2. The action of $S_{\infty}$ on itself by left multiplication extends to the action of $S_{\infty}$ on $\mathbb{N}^{\mathbb{N}}$ (the Baire space) by left composition. So instead of $\mathscr{F}\left(S_{\infty}\right)$, one can use $\mathscr{F}\left(\mathbb{N}^{\mathbb{N}}\right)$ and thus can take $\mathscr{F}\left(\mathbb{N}^{\mathbb{N}}\right)^{\mathbb{N}}$ as a universal space. But closed subsets of $\mathbb{N}^{\mathbb{N}}$ can be identified with trees on $\mathbb{N}$, i.e., subsets of $\mathbb{N}<\mathbb{N}$ closed under initial segments. A tree can be viewed now as a sequence of $n$-ary relations on $\mathbb{N}$, the $n$th relation identifying which $n$-tuples belong to the tree. Thus $\mathscr{F}\left(\mathbb{N}^{\mathbb{N}}\right)^{\mathbb{N}}$ can be embedded in the space of structures $X_{L}$, for the language containing infinitely many $n$-ary relation symbols for each $n$, and then by a simple coding pointed out by R. Dougherty, in the space of structures $X_{L_{\infty}}$ for the language $L_{\infty}$.

For Theorem 3.2. One first shows that the result holds for countable groups by adapting appropriately the proof of [N]. For an arbitrary $G$, let $G_{1}$ be a countable dense subgroup of $G$ and notice that if $G$ acts continuously, then any $G_{1}$-invariant measure is also $G$-invariant. Then apply Theorem 1.1 .

We would like to thank A. Louveau for many helpful suggestions concerning the results announced in this paper and G. Cherlin, who helped in improving considerably the exposition.

\section{REFERENCES}

[AM] L. Auslander and C. C. Moore, Unitary representations of solvable Lie groups, Mem. Amer. Math. Soc., vol. 62, Amer. Math. Soc., Providence, RI, 1966.

[Be] H. Becker, The topological Vaught's conjecture and minimal counterexamples, J. Symbolic Logic (to appear). 
[Bu] J. P. Burgess, Equivalences generated by families of Borel sets, Proc. Amer. Math. Soc. 69 (1978), 323-326.

[BM] J. P. Burgess and D. E. Miller, Remarks on invariant descriptive set theory, Fund. Math. 90 (1975), 53-75.

[C] G. Choquet, Lectures on analysis, W. A. Benjamin, New York, 1969.

[E] E. G. Effros, Transformation groups and $C^{*}$-algebras, Ann. of Math. (2) 81 (1965), 38-55.

[FHM] J. Feldman, P. Hahn, and C. C. Moore, Orbit structure and countable sections for actions of continuous groups, Adv. in Math. 28 (1978), 186-230.

[G] J. Glimm, Locally compact transformation groups, Trans. Amer. Math. Soc. 101 (1961), 124-138.

[HKL] L. Harrington, A. S. Kechris, and A. Louveau, A Glimm-Effros dichotomy for Borel equivalence relations, J. Amer. Math. Soc. 3 (1990), 903-928.

[K] A. S. Kechris, Countable sections for locally compact group actions, Ergodic Theory Dynamical Systems 12 (1992), 283-295.

[Ku] K. Kuratowski, Topology, vol. I, Academic Press, New York, 1966.

[L] D. Lascar, Why some people are excited by Vaught's conjecture, J. Symbolic Logic 50 (1985), 973-982.

[L-E] E. G. K. Lopez-Escobar, An interpolation theorem for denumerably long formulas, Fund. Math. 57 (1965), 253-272.

[Ma1] G. W. Mackey, Borel structures in groups and their duals, Trans. Amer. Math. Soc. 85 (1957), 134-165.

[Ma2] - Point realizations of transformation groups, Illinois J. Math. 6 (1962), 327-335.

[Ma3] Unitary group representations in physics, probability and number theory, AddisonWesley, Reading, MA, 1989.

[Mi] D. E. Miller, On the measurability of orbits in Borel actions, Proc. Amer. Math. Soc. 63 (1977), 165-170.

[Mo] C. C. Moore, Ergodic theory and von Neumann algebras, Proc. Sympos. Pure Math., vol. 38, Amer. Math. Soc., Providence, RI, 1982, pp. 179-225.

[N] M. G. Nadkarni, On the existence of a finite invariant measure, Proc. Indian Acad. Sci. Math. Sci. 100 (1991), 203-220.

[O] G. I. Olshanski, New “large groups" of type I, J. Soviet Math. 18 (1982), 22-39.

[Ra1] A. Ramsay, Topologies on measured groupoids, J. Funct. Anal. 47 (1982), 314-343.

[Ra2] Measurable group actions are essentially Borel actions, Israel J. Math. 51 (1985), 339-346.

[Ro] C. A. Rogers et al., Analytic sets, Academic Press, New York, 1980.

[Sa] R. Sami, Polish group actions and the Vaught Conjecture, Trans. Amer. Math. Soc. (to appear).

[Sil] J. H. Silver, Counting the number of equivalence classes of Borel and coanalytic equivalence relations, Ann. Math. Logic 18 (1980), 1-28.

[Sin] Ya. G. Sinai, Dynamical systems. II, Springer-Verlag, New York, 1989.

[St] J. R. Steel, On Vaught's Conjecture, Cabal Seminar 76-77, Lecture Notes in Math., vol. 689, Springer-Verlag, New York, 1978, pp. 193-208.

[U] V. V. Uspenskii, A universal topological group with a countable base, Funct. Anal. Appl. 20 (1986), 160-161.

[Var] V. S. Varadarajan, Groups of automorphisms of Borel spaces, Trans. Amer. Math. Soc. 109 (1963), 191-220.

[Vau] R. Vaught, Invariant sets in topology and logic, Fund. Math. 82 (1974), 269-294.

[Ve] A. M. Vershik, Description of invariant measures for the actions of some infinite-dimensional groups, Soviet Math. Dokl. 15 (1974), 1396-1400.

[VF] A. M. Vershik and A. L. Fedorov, Trajectory theory, J. Soviet Math. 38 (1987), 1799-1822. 
[Wh] V. M. Wagh, A descriptive version of Ambrose's representation theorem for flows, Proc. Indian Acad. Sci. Math. Sci. 98 (1988), 101-108.

[Wn] S. Wagon, The Banach-Tarski paradox, Cambridge Univ. Press, Cambridge and New York, 1987.

[Za] P. Zakrzewski, The existence of invariant probability measures for a group of transformations, preprint, July 1991.

[Zi] R. Zimmer, Ergodic theory and semisimple groups, Birkhäuser, Basel, 1984.

Department of Mathematics, University of South Carolina, Columbia, South CarOLINA 29208

E-mail address: becker@cs.scarolina.edu

Department of Mathematics, California Institute of Technology, Pasadena, CaliFORNIA 91125

E-mail address: kechris@romeo.caltech.edu 